\title{
Analysis of Devices to Detect Longitudinal Tear on Conveyor Belts
}

\author{
Alexander Zakharov ${ }^{1}$, Boris Geike ${ }^{1,2}$, Alexander Grigoryev ${ }^{1}$, and Alla Zakharova ${ }^{1}$ \\ ${ }^{1}$ T.F. Gorbachev Kuzbass State Technical University, 650000, Kemerovo, Russia \\ ${ }^{2}$ Institute of Coal at the Siberian Branch of the RAS, 650065, Kemerovo, Russia
}

\begin{abstract}
Abstarct. Currently mining enterprises more and more often use belt conveyors for mining operations. The length of transporting rock mass could be several kilometers, so in most cases conveyor are used. The transported rock mass is rather heterogeneous, as a rule, it has inclusions in a form of large pieces with sharp edges and, in some cases, metal objects, which might cause longitudinal tears of various lengths on conveyor belts. They lead to significant repair costs or replacement of expensive conveyor belt, loss of transported material, and downtime of the major enterprise equipment. In the world practice various devices and systems to detect longitudinal tears on conveyor belts have been developed for their successful operation. In this paper, there is analysis of existing devices to detect longitudinal tears on conveyor belts based on the use of mechanical (detection of the transported cargo on the lower the conveyor section and measuring the belt width), ultrasonic and electro-magnetic energy, evaluation of changes in the magnetic field configuration, computer vision, infrared laser and $\mathrm{x}$-ray radiation, image recognition software and embedded transponder chips.
\end{abstract}

\section{Introduction}

In the latest decades mining enterprises around the world practice utilization of a great number of continuous transports systems delivering minerals over considerable distances [1]. One of the most common devices for continuous transport is a belt conveyor characterizing by simple design, reliable operation and capacity to transport most types of bulk cargo. Technical and economic operating result of enterprises in the coal and mining industries mostly depends on the performance of conveyor belts and their economic indicators $[2,3]$.

Currently, underground transport is usually a conveyor line with 4 to 10 conveyors, and sometimes more. Failure of one conveyor causes shut down of the entire mine. The most severe consequences can be caused by a longitudinal tear on the conveyor belt. Economic cost to replace or repair belt (depending on the tear length) is added to the downtime cost of the major mine equipment. 


\section{Materials and Methods}

To reduce cost caused by these incidents, devices are being developed to detect the longitudinal tear on conveyor belts. The longitudinal tear detection system should promptly shut down a conveyor in the event of a belt tear to avoid a longer longitudinal tear.

Fixing the spillage of the transported cargo.

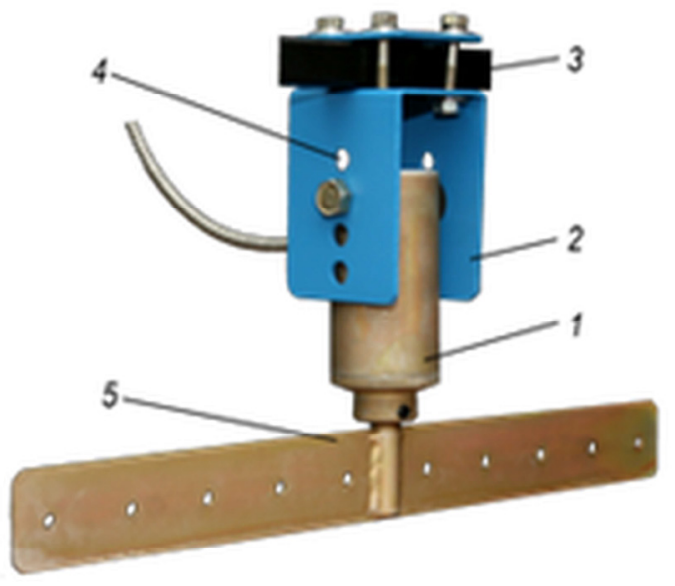

Fig. 1. LTCD Device to control material spillage.

One of the way to create devices for detecting longitudinal tear on a conveyor belt are devices for detecting spillage throughout a longitudinal tear. For example, longitudinal tear control detector LTCD (Fig. 1) is installed above the idle belt, attached by bracket 2 to the conveyor belting 3 . The bracket has holes for detector height adjustment 4 . In lower part of a detector there is a fixed extension cable with cross bar 5. The detector working position is vertical. In case of a longitudinal tear on the belt, the transported material is poured into the resulting hole from the working belt to the idle one, which carries it toward return pulley. When material reaches detector 1 , it acts on the bar and deflects it. The detector tilt on $20^{\circ}$ or more from the vertical changes its logical signal. A device LTCD is similarly arranged and working in package with inductive detectors or reed sensors.

Belt width measurement.

Another type of devices to detect longitudinal tear on the conveyor belt is a device that continuously measures the belt width. EMSYS is one of deviss made by MESKOTEX KG firm [4]. It is a compact and reliable control unit for belt conveyors, designed to meet the requirements of underground mining operations.

EMSYS control device to detect the belt longitudinal tear (Fig. 2) consists of: a station with two sensors to determine belt position; one double sensor roller; a base support; a control unit StG- 300- 02.

The control device to detect the belt longitudinal tear is installed directly nearby the standard grooved roller supports of the belt conveyor. The control unit interprets an invalid (positive or negative) change in the width of the belt as a longitudinal tear on the belt. Besides, the width and position of the conveyor belt is continuously monitored by the axial deviation of the detectors from the belt position, and their values are compared with the values of the profile and width of the belt embedded into the control unit. The belt profile is saved in the control unit during the training cycle. The training cycle ends automatically when the corresponding mark is passed. 


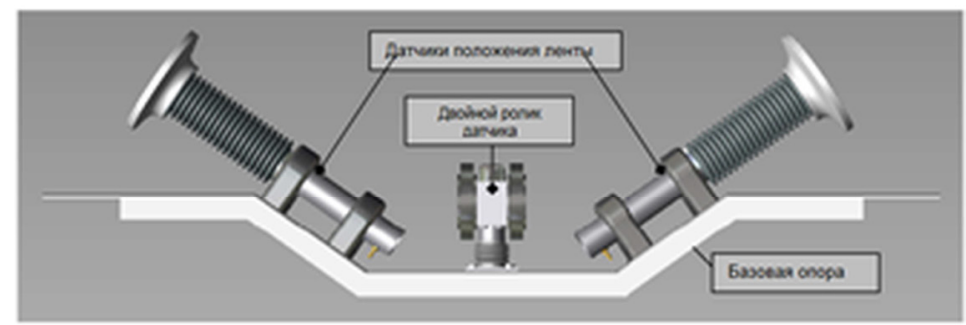

Fig. 2. EMSYS control device to detect the belt longitudinal tear.

The BG5k belt rip detector, Beltscan [4] provides non-contact control of the belt width by two ultrasonic probes constantly measuring the belt width. When a longitudinal belt rip occurs, the torn sections of the belt commonly move away from each other, or bend and fold over each other due to the angle and centering forces of the wing idler rollers. Should either of these two events take place, the width of the conveyor belt will change and a belt rip signal will be generated, which energizes the halt relay.

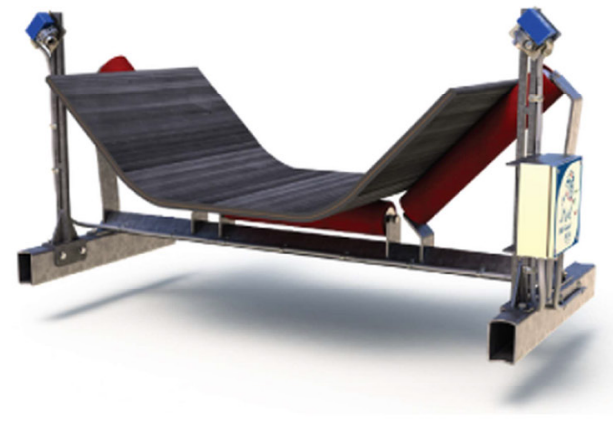

a)

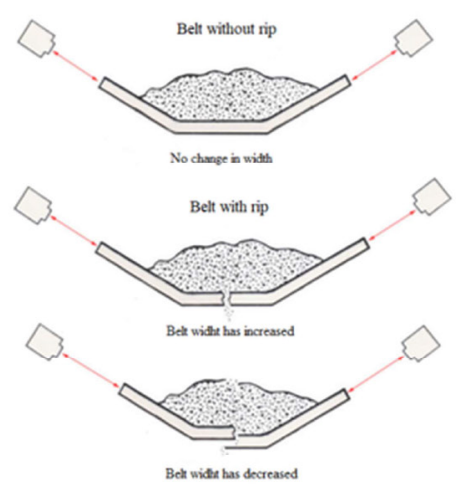

б)

Fig. 3. The BG5k belt tear detector, Beltscan; a) installation on conveyor, b) detector operating principle.

The ultrasonic probes BGlOk, Beltscan [4] (Fig. 4) should be positioned on opposite sides of the belt and be adjusted to use the belt edges as targets of measurements. The distance from a probe to the belt edge influences the device capacity, and is recommended to be $200 \mathrm{~mm}$, and this is considered efficient working distance for the conveyor.

The longitudinal rip probes operates on the principle of emitting ultrasonic energy into the conveyor belt at the control point. The energy is entered by a transmitter-converter and measured by several receivers-converters located on the opposite side of the belt. When a longitudinal rip occurs between the converters, level of acoustic energy entering the receiver is either reduced or completely lost. This reduction in energy level is used to detect a rip. 


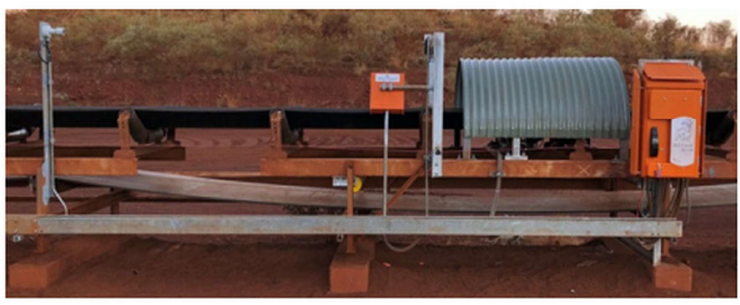

a)

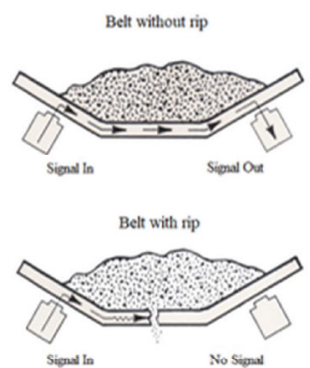

б)

Fig. 4. Probe BG10k, Beltscan; $a$ ) installation on conveyor, $b$ ) operating principle.

With some types of conveyor belts, the device does not work well enough, so Beltscan recommends sending a small sample (1000mm from the full width of the belt) of the conveyor belt for special probe settings. It should be noted that during operation of the conveyor belt, it gets damaged, including from fatigue stresses, and in places of the repaired belt serious deviations in sound conductivity are possible, and consequently, failures in the device operation.

Evaluation of the magnetic field configuration.

There are known devices to monitor a longitudinal tear on belts based on measuring magnetic field configuration (Fig. 5). The operating principle of such devices is as follows: a mesh grid of magnetized elements in a form of strips (rubber ferrite) or a magnetic matrix is embedded in the belt $[6,7,8]$. A special device takes pictures and remembers the magnetic field configuration. After each passage of the magnetic mesh grid over the control device, the existing configuration (current) is compared with the original fixed configuration. Should the belt is damaged in the place where the grid is located, the magnetic field configuration changes and after evaluation (comparison), the conveyor is shut down.

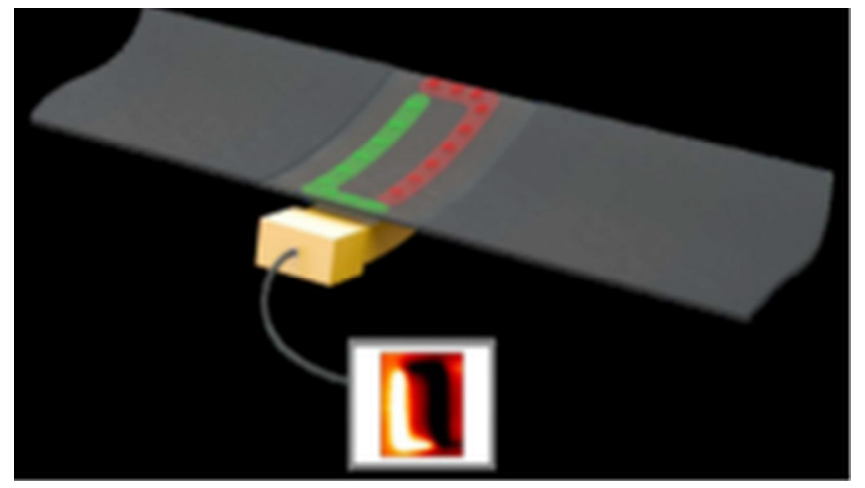

Fig. 5. Device with embedded magnetic rigid mesh in the conveyor belt.

Electromagnetic interaction.

Becker Mining BRS devices of various modifications [9], such as the ACS-A1 control system of Coal Control company [10], and other longitudinal rip detection systems applying principle of electromagnetic energy transmitting throughout a conveyor belt, 
have become quite widespread. Receiver and transmitter are installed on the conveyor belt against each other at a certain distance (Fig. 6).

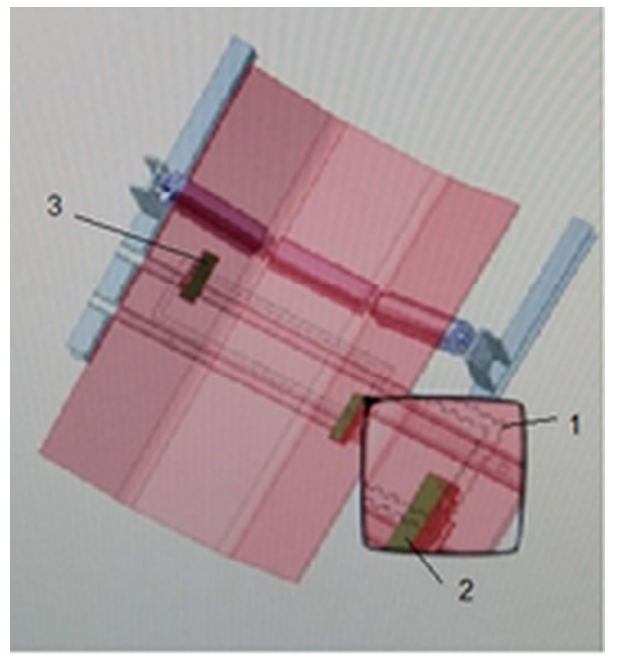

Fig. 6. Location of the transmitter and receiver inductive loops on the belt conveyor.

The transmitter sends an uninterrupted signal transmitting it to the receiver only when the loop is passed. The loop is vulcanized in the conveyor belt in such a way that at a certain moment there is a connection between the receiver and the transmitter be means of this loop. After the receiver has received the signal, it is transmitted further to the main system and processed. With a sufficient signal, the system continues to function under normal mode, and the described process is repeated when passing each inductive loop vulcanized in the belt. Should there no signal in the main system the conveyor belt immediately stops and an error message is displayed on the display of the computing unit. The large display makes it easier to maintain and collect system data. Due to the selfdiagnostic system, the most important errors are displayed directly on the display allowing a user to maintain service easily. To solve the issue of positioning, you should use software that calculates time of passing the loop relative to the transmitter and receiver. Adjusting the belt speed, this task becomes significantly more complicated that leads to a decrease in the probability of correct operation.

Computer vision, optics and sound, infrared, laser radiation.

Another way of creating devices to detect longitudinal tear on the conveyor belts is to analyze the image of its surface. It became possible with the advent of 'computer vision' software and digital photo i.e. the video equipment [11]. For auxiliary technologies, computer software to recognize image focused on special items have appeared $[12,13]$. In addition, with the growth of productivity and development of the automation systems in mining enterprises, environmental monitoring systems have appeared [14]. In particular, to increase the conveyor belts service life due to their timely repair, the systems to monitor the condition of conveyor belts came to operation, which should record their damage, including longitudinal tear [15].

One of the ways to get an image is to use laser radiation. A red band of laser radiation is projected onto the surface of the conveyor belt, and the image obtained by the appropriate CMOS image sensor is processed and analyzed.

There is also an integrative method to detect a tear by reproducing binocular vision based on infrared and visible radiation; as a result, a longitudinal rip on the conveyor belt could be detected 
A dual-band infrared detection method based on the ranges of mid-infrared and longinfrared radiation is also used for the conveyor belts longitudinal tear detection.

A multispectral method for visual detection of longitudinal tear on the conveyor belt has been tested in the industrial sphere. The experiment results prove that the multispectral visual detection method can not only identify the longitudinal tear on the conveyor belt, but also accurately classify and identify other status of the conveyor belt. The accuracy of the multispectral visual detection method is $90.06 \%$, and the recognition accuracy of the longitudinal tear is $92.04 \%$.

An audio-and-visual detection method for a longitudinal tear on the conveyor belt is known. A video camera and microphone system are used to collect images and audio signals from the conveyor belt, and damages are detected by both the image and sound, respectively. Then the results of image and sound analysis are combined.

X-radiation.

Phoenix has developed the Phoenoguard ${ }^{\circledR}$ PX system for continuous monitoring of the conveyor belt condition, including longitudinal rips, based on the X-rays. The transmitting and receiving devices to monitor the conveyor belt status are mounted in a single unit, which is usually located on the empty belt of the conveyor.

Embedded microchips - transponders.

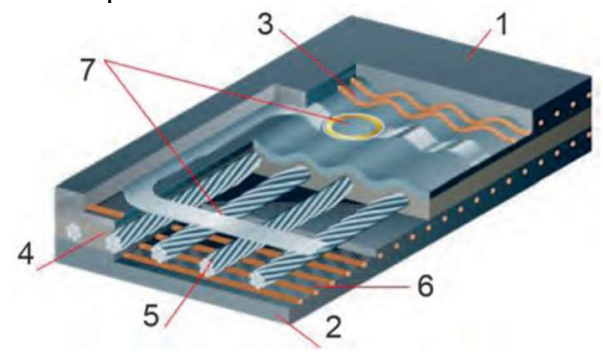

Fig. 8. Special type of conveyor belt ContiTronic $\mathbb{R}$ to control its status: 1 - top conveyor cover; 2 bottom conveyor cover; 3 - upper protective cross-section, synthetic cables; 4 - frame of rubbercable belt; 5 - steel cables of belt frame; 6 - lower protective cross-section, synthetic cable; 7 instrumentation-microchip transponder.

In order to detect a longitudinal tear on the conveyor belts, a special type of conveyor belt ContiTronic $\AA$ has been developed [25]; inside this belt special transponders are vulcanized at a certain interval along the length of the belt, (Fig. 8), by means of the transponders the required data is transmitted to the receiver, and subsequently to the computer, where they are analyzed and displayed on display

\section{Discussion}

In the event of a through longitudinal tear on the conveyor belt, the transported cargo will spill onto the empty belt. There are several designs of devices to detect the spillage, featuring simplicity and low cost. However, it should be noted, should parts of the load fall onto the empty belt when there is a belt drift, or for other reasons, a false alarm may occur.

Control of the belt width by EMSYS system requires a lot of equipment and is rather complicated, and it does not exclude a possibility of failure in case of a longitudinal tear with a thin object. Control by BG5k ultrasound is easier, but during operation there is a possibility of a local width change and configuration of the belt edge.

The use of the conveyor belt as an ultrasound conductor in terms of constructability is not difficult, but in case of the conveyor belt repair during operation, it is possible to violate 
the ultrasonic energy conductivity and malfunction, since each type of the conveyor belt requires its own settings.

Evaluation of the magnetic field configuration requires complex equipment and complex integration of the magnetized matrix into the belt; furthermore, there is a possibility of altering the magnetization of its individual elements from the shock load impact at the loading point of the belt conveyor.

Electromagnetic energy transmission to detect a longitudinal tear is possible with an embedded conductive loop into the conveyor belt, what causes certain difficulties during its installation; furthermore, in this loop, due to the large number of pickups in it, there could be difficulties with reading information by the receiver, so, there is a need to shield the transmitter, thus the reliability of operation decreases.

Advantage of the systems applying image analysis of the conveyor belt surface utilizing machine vision, infrared and x-ray technologies means that there is no need to change a design of the conveyor belts. However, operation of the optical devices in a dusty environment (underground workings) is very problematic. Besides, the level of the recognition accuracy will decrease with the change in the conveyor belt surface condition and with an increase of its operational time.

As for the use of x-rays and embedded into the belt transponders it should be noted that such settings can be applied only for the empty belt of the conveyor, and this eliminates the efficiency of the conveyor shutdown in case of the throughout rip of the belt.

\section{Conclusion}

The longitudinal tear on the conveyor belt is a significant problem in the world practice of the belt conveyors operating. There is quite a large number of various devices for detecting longitudinal rips. Some of them, such as evaluation of the magnetic field configuration, transmission of the electromagnetic induction, and utilization of the transponders, require changes to the design of the conveyor belt. Others, such as the use of ultrasound and multispectral visual detection, significantly depend on the belt service life, i.e. when the belt accumulates areas that have been repaired; the reliability of the systems is reduced. It should be highlighted as a promising direction with the use of electromagnetic energy. These systems do not depend on the service life, and the embedding of conductive loops is possible at the belt manufacture.

\section{References}

1. M. Thompson, A. Jennings, Min., 14 (2016)

2. F. Hakami, A. Pramanik, N. Ridgway, A. K. Basak, Tribol. Int., 111 (2017)

3. T. Braun, A. Hennig, B.G. Lottermoser, J. Sustain. Min., 16 (2017)

4. R. Blazej, L. Jurdziak, A. Kirjanow, T. Kozlowski, Diagnostyka, 16, 3 (2015)

5. Y. Pang, G. Lodewijks, 2006 IEEE Int. Conf. Serv. Oper. Logist. Informatics, SOLI, 803 (2006)

6. R. Błażej, L. Jurdziak, T. Kozłowski, A. Kirjanów , Meas. J. Int. Meas. Confed., 123 (2018)

7. M. Leo, G. Medioni, M. Trivedi, T. Kanade, G.M. Farinella, Comput. Vis. Image Underst., 154 (2017)

8. G. Rosati, G. Boschetti, A. Biondi, A. Rossi, Opt. Lasers Eng., 47 (2009)

9. Y. Huang, W. Cheng, C. Tang, C. Wang, Ecol. Indic., 51 (2015) 
10. A. Harrison, 21st int. Conf. of Safety in Mines Research Institutes, 3, 3 (1985)

11. L. Xianguo, S. Lifang, M. Zixu, Z. Can, J. Hangqi, Optik (Stuttg), 168 (2018)

12. T. Qiao, L. Chen, Y. Pang, G. Yan, and C., Measurement, 110 (2017)

13. B. Yu, T. Qiao, H. Zhang, G. Yan, Meas. J. Int. Meas. Confed., 120 (2018)

14. C. Hou, T. Qiao, H. Zhang, Y. Pang, and X. Xiong, Meas. J. Int. Meas. Confed., 143 (2019)

15. C. Hou, T. Qiao, M. Qiao, X. Xiong, Y. Yang, H. Hhang, IEEE Access., 7 (2019) 